Sparse graph codes for the two-way relay network with correlated sources

By Gottfried Lechner, Roy Timo, \&Lawrence Ong

Copyright (C) 2011 IEEE.

This is an author-prepared version of the paper, reprinted from the Proceedings of the IEEE Data Compression Conference, DCC 2011, p. 466.

http://dx.doi.org/10.1109/DCC.2011.32

This material is posted here with permission of the IEEE. Such permission of the IEEE does not in any way imply IEEE endorsement of any of University of Newcastle's products or services. Internal or personal use of this material is permitted. However, permission to reprint/republish this material for advertising or promotional purposes or for creating new collective works for resale or redistribution must be obtained from the IEEE by writing to pubs-permissions@ieee.org. By choosing to view this document, you agree to all provisions of the copyright laws protecting it. 


\title{
Sparse Graph Codes for the Two-Way Relay Network with Correlated Sources
}

\author{
Gottfried Lechner ${ }^{1}$, Roy Timo ${ }^{1}$, and Lawrence Ong ${ }^{2}$ \\ ${ }^{1}$ Institute for Telecommunications Research, University of South Australia \\ ${ }^{2}$ School of Electrical Engineering and Computer Science, The University of Newcastle \\ E-mail: \{gottfried.lechner, roy.timo\}@unisa.edu.au, lawrence.ong@cantab.net
}

We consider the two-way relay network where two nodes communicate via a relay. We assume that the data at the nodes are correlated (e.g., measurements in a sensor network) and that there is no direct communication between the nodes. The nodes communicate via the relay using a two-phase protocol consisting of an uplink part over an orthogonal multiple access channel and a downlink part over a broadcast channel.

The individual codes as well as the overall system can be represented by a joint factor graph consisting of a source code at each node, a channel code for the each uplink and a channel code for the downlink. The optimality of separation of source and channel coding [1] implies that it is optimal to individually design these codes. We focus on low-density parity-check codes where code design corresponds to the optimisation of their degree distributions [2].

We assume correlated, memoryless binary messages represented by the vectors $\mathbf{W}^{(1)}$, $\mathbf{W}^{(2)}$ of length $N_{s}$ where the elements are taken iid from a joint distribution $q\left(w^{(1)}, w^{(2)}\right)$. In the uplink phase, the nodes encode their respective messages to binary codewords $\mathbf{X}^{(1)}$, $\mathrm{X}^{(2)}$ of length $N_{c}$. The bandwidth expansion factor is defined as $\kappa \triangleq N_{c} / N_{s}$, i.e., as the number of channel uses per source symbol. Necessary and sufficient conditions for the achievability of $\kappa$ are given [1].

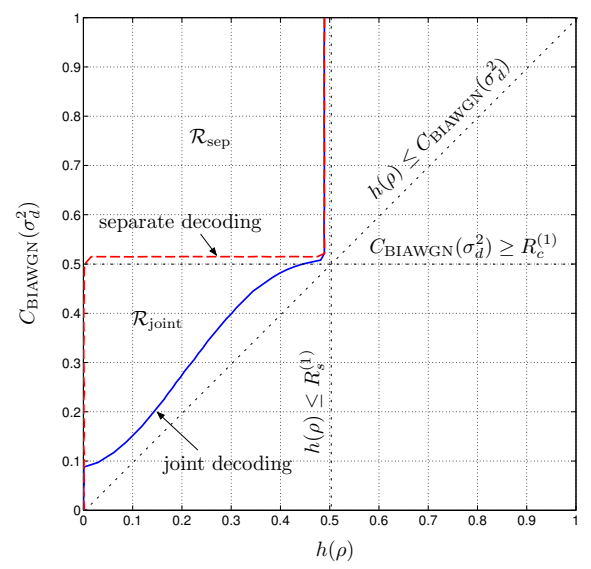

While the channel decoder at the relay is a standalone decoder, there are two options at the nodes: i) separate decoding or ii) joint decoding of the relay message and the other node's message.

As an example we assume uniform sources correlated via a BSC with cross-over probability $\rho$ and reliable communication on the uplink channels. The broadcast channel is a Gaussian channel with variance $\sigma_{d}^{2}$. Furthermore, the rate of the source and channel codes is $1 / 2$ and $\kappa=1$. We are now interested in the pairs $\left(\rho, \sigma_{d}^{2}\right)$ which define the achievable region $\mathcal{R}$ where the iterative decoder converges.

When non capacity-achieving codes are applied (or the sources have a stronger correlation than designed for) then a joint decoder can exploit the remaining redundancy in the source and outperforms a separate decoding scheme.

[1] R. Timo, L. Ong, and G. Lechner, "The two-way relay network with arbitrary correlated sources and an orthogonal MAC," in Proc. Data Compression Conference (DCC), 2010.

[2] T. J. Richardson and R. Urbanke, Modern Coding Theory. Cambridge University Press, 2008.

The work of G. Lechner and R. Timo was funded by the Australian Research Council Grants DP0881160 and DP0880223. The work of L. Ong was funded by the Australian Research Council Grants DP0877258 and DP1093114. 